\title{
Los wixáritari con diabetes mellitus y sus vínculos con la enfermedad: desde la aparición del síntoma hasta una primera explicación
}

\author{
Wixáritari with diabetes mellitus and their links with the \\ disease: From the appearance of the symptom to a first \\ explanation
}

Francisco Quiñonez-Tapia' ${ }^{1}$ María de Lourdes Vargas-Garduño², Rubén Soltero-Avelar ${ }^{3}$

${ }^{1}$ Doctor en Psicología. Profesor de tiempo completo, Universidad de Guadalajara, Jalisco, México. $₫$ iD

${ }^{2}$ Doctora en Ciencias Sociales. Profesora de tiempo completo, Universidad Michoacana de San Nicolás de Hidalgo, Michoacán, México. $₫$ (iD

${ }^{3}$ Doctor en Ciencias de la Salud Pública. Profesor de tiempo completo, Universidad de Guadalajara, Jalisco, México. $\bowtie$ iD
RESUMEN Se recuperó el proceso de construcción del esquema conceptual, referencial y operativo (ECRO) de los wixáritari sobre la diabetes mellitus tipo 2 en una comunidad de Jalisco, México, desde la aparición del primer síntoma hasta una posible explicación de la enfermedad. Se realizó un estudio cualitativo con el método fenomenológico a partir de entrevistas en profundidad para la recolección de información. Se contó con siete participantes. Los datos se procesaron con el método de análisis del discurso de Bajtín, a partir de la teoría del vínculo de Pichon-Rivière. Se encontró que el wixárika con diabetes construye su esquema conceptual, referencial y operativo durante un periodo de tres a cinco años, a partir de tres vínculos: los síntomas en el cuerpo, la explicación del wixárika a sus síntomas, y las explicaciones del espacio social a su enfermedad. El wixárika construye una serie de vínculos y un esquema conceptual, referencial y operativo que le permite validar una explicación de lo que experimenta en el cuerpo, en medio de un proceso confrontativo y doloroso, Ileno de afirmaciones y negaciones hasta llegar a una síntesis, que le permita comprender su situación e instrumentar una conducta para el cuidado de su salud. PALABRAS CLAVES Diabetes Mellitus Tipo 2; Salud de Poblaciones Indígenas; México.

\begin{abstract}
This article explores the process of construction of the conceptual, referential and operative schema among the wixáritari population regarding type 2 diabetes mellitus in a community of Jalisco, Mexico, from the appearance of the first symptom to a possible explanation of the disease. This is a qualitative study performed with the phenomenological method using in-depth interviews to collect information. There were seven participants. The data was processed with Bakhtin's discourse analysis, based on Pichon Rivière's theory of the link. It was found that the wixáritari population with diabetes develop their conceptual, referential and operative schema over a period of three to five years, based on three links: the symptoms in the body, the explanation the wixáritari give regarding their symptoms, and the explanations of the social space of the disease. The wixáritari develop a series of links and conceptual, referential and operative schema that allow them to validate an explanation of what they experience in their body, in a confrontational and painful process full of affirmations and denial until reaching a synthesis that allows them to achieve a full understanding of their situation and implement behaviors to care for their health.
\end{abstract}

KEY WORDS Diabetes Mellitus, Type 2; Health of Indigenous Peoples; Mexico. 


\section{INTRODUCCIÓN}

La Organización Mundial de la Salud, en mayo de 2012, alertó acerca del aumento de casos de diabetes ${ }^{(1)}$. El Programa de las Naciones Unidas para el Desarrollo, en el año 2010, señalaba que más de la mitad de indígenas mayores de 35 años padecían diabetes mellitus tipo 2, lo que implicaba que esta enfermedad ponía en peligro la existencia de algunas comunidades indígenas del planeta. Asimismo, mencionaba que en cuestión de diabetes no se encontraron discrepancias entre la proporción de casos de diabetes en indígenas y no indígenas ${ }^{(2)}$. Por lo tanto, las comunidades indígenas de todo el mundo no están ajenas a la dinámica epidemiológica de esta enfermedad.

Los wixáritari (en su lengua originaria, se usa el término "wixárika" para el singular, y "wixáritari", para el plural) del norte de Jalisco, México, presentaron una frecuencia de 22 casos nuevos en el 2008 y para el 2013 ya se tenían 125 nuevos casos: esto refleja un $568 \%$ de aumento en la frecuencia de la diabetes ${ }^{(3)}$. Este aumento se registró mediante el protocolo de diagnóstico de la diabetes que incluyó la historia clínica completa, la evaluación de familiares de primer grado que padecieran la enfermedad y pruebas de laboratorio, tales como: glucosa plasmática al azar, glucosa en plasma en ayunas, glucosa en plasma a las 2 horas y con la prueba de hemoglobina glucosilada $(\mathrm{HbA} 1 \mathrm{c})^{(4)}$. De las 483 localidades wixáritari, destaca la comunidad de Mesa del Tirador de Bolaños, Jalisco, cuya población de 767 habitantes $^{(5)}$ fue la que presentó mayor prevalencia de casos nuevos de diabetes tipo 2 durante el periodo 2008- 2013 (de 1 a 21 casos), en el grupo de edad de 24 a 45 años, seguido del grupo de edad de 65 y más ${ }^{(3)}$. Esto sucede, muy probablemente, debido a su cercanía con poblaciones mestizas, cuyo régimen alimenticio rico en carbohidratos y azúcares influye en la modificación de sus hábitos tradicionales y los predisponen al desarrollo de la diabetes mellitus tipo $2^{(6,7,7,9,10,11,12,13)}$, como es el caso de la adopción, a su dieta regular, de alimentos industrializados tales como la sardina, atún, galletas, frijoles y elote enlatados, salchicha, puré de jitomate, jamón, pastas, lácteos procesados (yogur, queso, leche), cereales, refresco (Coca-Cola) dulces altos en azúcar, pan dulce, cerveza, tequila, mayonesa, sopas instantáneas, bebidas energéticas y jugos, por mencionar algunos. Dichos alimentos modificaron la dieta tradicional de los wixáritari, basada en el maíz, frijol, calabaza y caldos de venado, becerro, gallina, pescado o iguana ${ }^{(14,15,16,17)}$. Por ende, las localidades con mayores dificultades de acceso a poblados mestizos no presentan casos de diabetes.

El pueblo wixárika se ubica en la Sierra Madre Occidental de México ${ }^{(18)}$ y, de acuerdo con la Comisión Nacional para el Desarrollo de los Pueblos Indígenas ${ }^{(19)}$, en el país existe una población de 59.820 habitantes. Con relación a la comprensión de los procesos de salud-enfermedad, para los wixáritari "existen dos tipos de enfermedades: las de la sierra y las traídas por los españoles"(20), y de acuerdo con los usos de la medicina tradicional, se considera que "todos los elementos de la naturaleza constituyen la farmacia"(20), por lo que el conocimiento y la aplicación de las propiedades de la naturaleza en su vida cotidiana ha sido clave en la persistencia de los wixáritari ${ }^{(21)}$.

El wixárika concibe la enfermedad como una falta de responsabilidad hacia las deidades, un mal perpetrado o algo que extravió el alma (kupuri $^{(22)}$. Asimismo, la enfermedad y la muerte, en la cultura wixárika, se atribuyen a causas como: un castigo de los dioses por quebrantar un deber religioso, una señal de que no se cuenta con el favor de los dioses, una falta de fuerza o valor que hace que la víctima caiga presa de malos pensamientos o por magia negra ${ }^{(21)}$.

La curación de las enfermedades de los wixáritari es realizada por los mara'kates cantores, quienes ejecutan sus milagros de profecía y curación mediante el canto de los mitos y la prestidigitación; o simplemente por curaciones, sin $\operatorname{cantos}^{(23)}$. La curación es una lucha en la que el poder de los mara'kates se enfrenta con el de aquel que 
"se ha posesionado del enfermo"(23). Además, en las comunidades wixáritari existen centros de salud dependientes del Estado mexicano (Sistema Nacional de Salud), que aplica programas, intervenciones y tratamientos para la prevención y control de la diabetes. Asimismo, de los 59.820 habitantes que conforman el pueblo wixárika, 37.046 están inscritos en el Sistema Nacional de Salud, 22.205 no lo están y no se tiene registro de la situación de los 569 restantes ${ }^{(19)}$.

La Secretaría de Salud cuenta con 21 unidades de atención para la salud de los wixáritari: doce centros y nueve módulos de salud, localizados en las 483 poblaciones indígenas de los municipios de Bolaños y Mezquitic, Jalisco, donde se encuentran cuatro de los cinco gobiernos tradicionales (San Andrés Cohamiata, San Sebastián Teponohuaxtlán, San Miguel Huaixtita y Tuxpan de Bolaños) ${ }^{(24)}$.

A pesar de ello, la prevalencia de la diabetes se sigue incrementando en la población wixárika, lo cual evidencia la necesidad de diseñar e implementar estrategias complementarias que permitan incidir de manera más significativa en el control de esta enfermedad, dado que implica un cambio de mentalidad, la modificación de hábitos, los usos y costumbres y la generación y transferencia de conocimientos en torno a los procesos de salud-enfermedad para comprender las conductas que generan la elección o no del tratamiento ${ }^{(25)}$. De ahí la importancia de estudios psicosociales como el que se presenta en este texto, que brindan información relevante para fundamentar dichas intervenciones. Es necesario señalar que numerosos estudios realizados en poblaciones indígenas ${ }^{(26,27,28,29,30)}$, desde abordajes teóricos de la antropología, de la fenomenología, de la economía o el enfoque sanitario, muestran que uno de los factores más importantes en la relación con el sistema de salud público depende de cuán discriminados o aceptados se sientan por parte de los efectores de salud. Además, para favorecer el cumplimiento de las prescripciones a los tratamientos se requiere del conocimiento de los saberes y prácticas populares ${ }^{(31)}$.

Otros estudios han reportado que el tipo de experiencias (positivas o negativas) que se generan y los estilos de comunicación médico-paciente que se utilizan en el modelo biomédico determinan la comprensión o no de la enfermedad y la adherencia o no al tratamiento ${ }^{(32,33,34,35,36,37)}$. De allí la necesidad de promover, en los médicos, el desarrollo de actitudes que permitan establecer relaciones de confianza con los pacientes indígenas y de medios rurales, partiendo de la comprensión de su cosmovisión, para adecuar los conceptos biomédicos e integrarlos a la subjetividad de los indígenas, y evitar que el personal de salud asuma actitudes agresivas que culpabilicen a los pacientes de su condición de salud ${ }^{(38,39)}$. Por lo tanto, en este texto se propone un fundamento teórico innovador, basado en el psicoanálisis social de Pichón Rivière, para generar estrategias que permitan el acercamiento cultural médico-paciente, puesto que como dice Whitty-Roger, cuando la comprensión del cuerpo con diabetes, desde el modelo biomédico se ve entorpecida, genera en los pacientes, sentimientos de angustia, incertidumbre, miedo, falta de energía, sufrimiento, sensación de pérdida de control, disminución de la energía, malestar, entre otros $^{(39)}$. De ahí la importancia de este estudio que pretende describir la modificación de la subjetividad de los wixáritari que padece diabetes mellitus tipo 2 , desde la aparición de síntoma hasta una primera explicación con base en el modelo de Pichón Rivière.

\section{Desarrollo teórico}

Para el logro del objetivo, se ha fundamentado teóricamente el estudio en el psicoanálisis social desde la teoría del vínculo(40). A partir de la propuesta de Byron $\operatorname{Good}^{(41,42)}$ se consideró pertinente explorar algunas propuestas que permitieran una comprensión más profunda de la experiencia vivida por los wixáritari con la diabetes mellitus tipo 2 , para de este modo, proponer estrategias que faciliten la comunicación entre médicopaciente. Al respecto, la teoría de Pichon-Rivière permite dicho acercamiento. Esta teoría sostiene que el sujeto está constituido por dimensiones intrasubjetivas e intersubjetivas 
relacionadas dialécticamente y articuladas en una totalidad por tres dimensiones: la mente (área 1), el cuerpo (área 2) y el exterior (área $3)^{(40)}$. Asimismo, el sujeto se constituye por lo exterior y lo interior, en medio de las tensiones y contradicciones que genera la interacción entre lo individual y lo social, entre lo intersubjetivo y lo intrasubjetivo, y es en medio de esa dialéctica donde también se constituye la experiencia frente al fenómeno vivido, en una historia y cultura particular ${ }^{(43)}$.

De acuerdo con ello, la subjetividad es un sistema abierto en constante estructuración, que se construye de manera dialéctica entre la estructura social e individual, entre los elementos históricos, individuales y sociales del sujeto, y se configura de adentro hacia afuera y de afuera hacia dentro, en medio de tensiones y contradicciones ${ }^{(40,43,44)}$. Así, la subjetividad está constituida por un conjunto de vínculos tanto externos como internos que establece el sujeto con los objetos, proyectados o introyectados desde los campos de interacción en los que se encuentren, en un tiempo y espacio específicos ${ }^{(40)}$.

El vínculo es una "estructura dinámica y compleja de relación que incluye a un sujeto y un objeto en interacción dialéctica, su determinación es recíproca en un proceso de comunicación y aprendizaje que tiene como resultado una conducta con ese objeto"(40), y es particular para cada sujeto y momento histórico y cultural, lo que le permite insertarse en el campo simbólico del grupo al que pertenece $^{(43)}$.

Pichon-Rivière ${ }^{(40)}$ plantea la existencia del campo psicológico: interior o exterior. Es en el campo psicológico donde los objetos pueden estar situados y establecer dos tipos de vínculos: externos e internos. El sujeto y objeto establecen una relación dialéctica entre sujeto-mundo, individual-social, internoexterno, adentro-afuera; existe una conexión que hace posible la relación constante y permite establecer vínculos ${ }^{(40)}$. Al existir el campo psicológico, el sujeto puede establecer relaciones internas y externas con el objeto: al estar en un campo externo, puede ser introyectado al campo interno y trasferir la relación o el vínculo externo a un vínculo interno, una vez introyectado el objeto; al estar en el campo interno, puede ser llevado al campo externo a través de la proyección, y de esta manera pasar de una relación interna a una relación externa determinado objeto ${ }^{(43)}$.

Así también, el psicoanálisis social ${ }^{(43)}$ propone el esquema conceptual, referencial y operativo (ECRO), que se constituye por un entramado de vínculos sobre objetos específicos; por lo cual, cada esquema corresponde a objetos particulares. El ECRO es un conjunto articulado de conocimientos, referentes y conductas específicas sobre objetos con los cuales los sujetos entran en contacto en la realidad. Se caracteriza por ser un instrumento que permite aprehender la realidad, el cual puede ser transferible de generación en generación $^{(40)}$. El ECRO es una producción individual y social que nos permite dar cuenta de las condiciones de existencia, el conjunto de experiencias, conocimientos y afectos en los que un sujeto está inmerso, y genera en los sujetos modelos de sensibilidad, modos de pensar, sentir y hacer en el mundo y que marcan su existencia de una forma determinada ${ }^{(40,44)}$. En la Figura 1 se presenta de manera esquemática el ECRO, los vínculos y las áreas constitutivas del sujeto, aplicados al estudio del wixárika con diabetes.

En esta línea de pensamiento, puede decirse que el wixárika (sujeto) y la diabetes (objeto) están en una relación dialéctica, en la que el wixárika es construido por la diabetes, pero también la diabetes es construida por el wixárika. El wixárika establece la conexión subjetiva con la diabetes que padece, a partir de los distintos vínculos que genera. Esta conexión subjetiva (el vínculo) con la enfermedad es dinámica, ya que va cambiando constantemente en función de la experiencia vivida y de los significados en determinados espacios y tiempos, y modifica las interacciones con el grupo indígena al que pertenece.

La enfermedad crónica denominada diabetes, que es el objeto de conocimiento del indígena wixárika, puede estar situado tanto en el exterior como el interior. En el exterior (área 3: contexto sociocultural al que pertenece el indígena), construye socialmente subjetividades en torno a las enfermedades. 


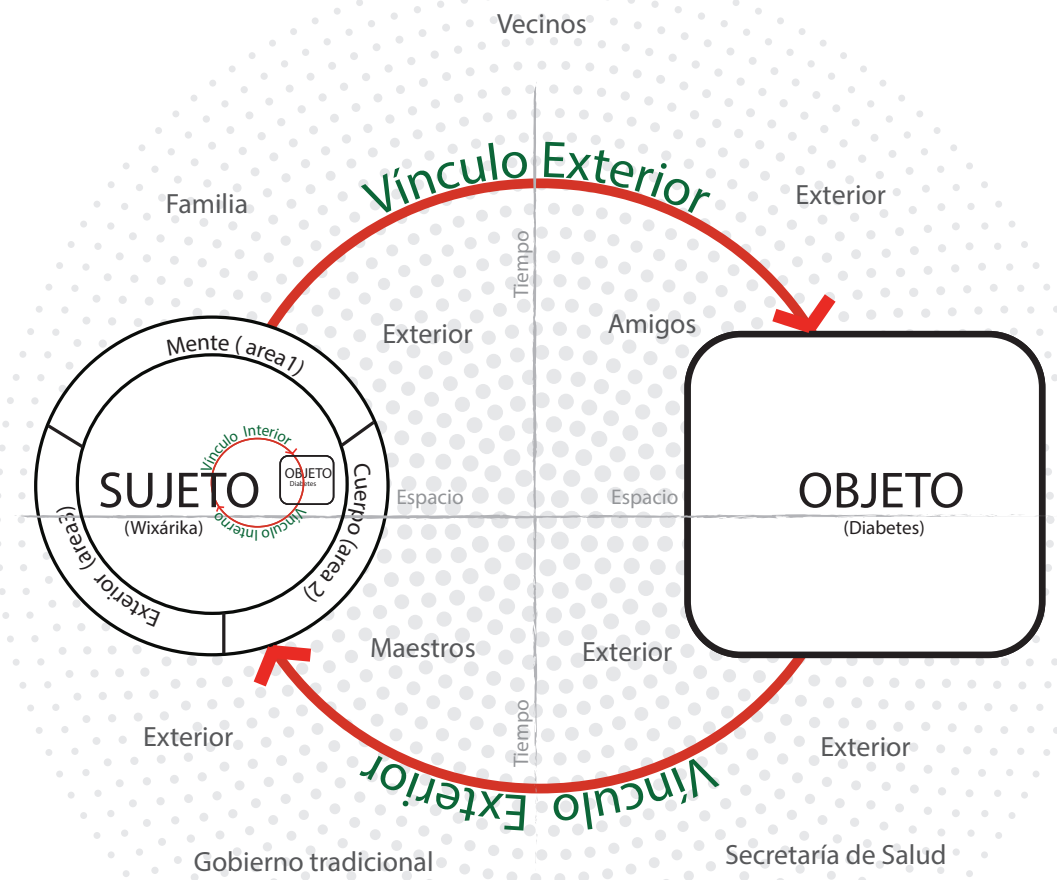

Mara'kame

Figura 1. Teoría del vínculo aplicada al estudio del wixárika con diabetes.

Fuente: Elaboración propia.

En el ámbito interno, es el sujeto indígena quien padece físicamente la enfermedad (área 2: cuerpo) y construye subjetividades individuales asociadas a sus creencias, en cuanto a su padecimiento (área: 1 mente). Con la interacción de lo externo y lo interno, se construyen los vínculos con el objeto (diabetes) en una relación dialéctica con el wixárika. Asimismo, es importante recuperar el ECRO que construye el wixárika sobre la diabetes, debido a que permite comprender las experiencias, conocimientos y las vivencias que construyen los wixaritari en torno a su padecimiento.

\section{MÉTODO}

El presente estudio forma parte de una investigación más amplia titulada "La subjetividad del wixárika en la experiencia vivida con la diabetes mellitus". El estudio se llevó a cabo desde el paradigma cualitativo ${ }^{(45)}$, se empleó el método fenomenológico crítico de Merleau-Ponty ${ }^{(46,47,48,49)}$ para la recuperación de los datos. El contexto de la investigación fue la comunidad indígena wixárika denominada Mesa del Tirador del municipio de Bolaños, Jalisco, México. Los criterios de inclusión de 
los participantes fueron: ser indígena wixárika, pertenecer a la comunidad indígena aludida, contar con un diagnóstico de diabetes mellitus tipo 2 por parte de la Secretaría de Salud y ser mayor de 25 años. Los criterios de exclusión: no tener disposición de participar en el estudio, no aceptar el tiempo requerido que demanda el método biográfico, no aceptar ir al lugar de encuentro propuesto. Adicionalmente, no formaron parte de la muestra final quienes decidieron desertar del estudio de manera voluntaria.

Se utilizó el muestreo teórico(50,51), por lo que con siete wixáritari se llegó al punto de saturación (Tabla 1).

Se empleó la técnica de entrevista en profundidad ${ }^{(52,53,54)}$. Se realizaron dos entrevistas por persona, con una duración de 60 a 90 minutos cada una, con un intervalo de 2 días. Las entrevistas se grabaron con consentimiento de los participantes y se transcribieron sin alterar los discursos. La primera entrevista fue en el domicilio de cada participante, donde se estableció el encuadre. La segunda entrevista se realizó en las instalaciones de la Secretaría de Salud. Para el análisis de los relatos de vida se utilizó el método de análisis del discurso de Bajtín ${ }^{(5,56)}$ y se utilizó el software de ATLAS.ti (versión 7) ${ }^{(57)}$.

Esta investigación se apega a los principios del Reglamento de la Ley General

Tabla 1. Caracterización de la muestra.

$\begin{array}{lccc}\text { Participantes } & \text { Edad } & \text { Ocupación } & \begin{array}{c}\text { Tiempo de padecer } \\ \text { diabetes tipo 2 } \\ \text { (años) }\end{array} \\ \text { Isela } & 27 & \text { Hogar } & 2 \\ \text { Rosa } & 70 & \text { Hogar } & 12 \\ \text { Juventino } & 78 & \text { Hogar } & 3 \\ \text { Claudia } & 85 & \text { Hogar } & 13 \\ \text { Leticia } & 57 & \text { Hogar } & 13 \\ \text { José } & 53 & \text { Tienda de abarrotes } & 18 \\ \text { Fátima } & 61 & \text { Hogar } & 14\end{array}$

Fuente: Elaboración propia.

Nota: La edad es considerada en años aproximados, debido a que ninguno de los participantes, cuenta con esa información. El tiempo de padecer diabetes mellitus tipo 2 es en años aproximados, de acuerdo con lo reportado por el/la participante. Los nombres fueron cambiados para salvaguardar su integridad. de Salud en Materia de Investigación para la Salud de México ${ }^{(58)}$ y al Código Ético del Psicólogo de México ${ }^{(59)}$. Por tanto, se solicitó la autorización a la Secretaría de Salud y al tatuwani (gobernador tradicional) de Tuxpan de Bolaños, Jalisco, para realizar la investigación en la comunidad Mesa del Tirador; además de contar con el consentimiento informado oral de los siete participantes. Asimismo, la investigación fue aprobada por un comité de ética de la Universidad de Guadalajara, que fundamenta sus decisiones en el Código Ético del Psicólogo y en el Reglamento de la Ley General de Salud en Materia de Investigación para la Salud, de México, aludidos al inicio de este párrafo; puesto que no se cuenta con un código institucional al respecto.

\section{RESULTADOS}

La narrativa del wixárika no se entreteje en una organización temporal medida en años, meses, semanas, días, puesto que, en su cultura, utilizan sucesos de vida para este fin. Por ende, los participantes establecieron contacto con la diabetes cuando se presentó la sintomatología en el cuerpo. Este momento histórico marca el inicio de un encuentro cuyo resultado es la producción de un sentido subjetivo particular del wixárika hacia la diabetes.

En consecuencia, este momento de análisis inicia desde la aparición de lo que está ahí y que existe en el cuerpo (el síntoma diabético) hasta una posible explicación de lo que experimenta. Para comprender el encuentro del wixárika (sujeto) con la diabetes (objeto), se generaron tres categorías de análisis: a) los síntomas en el cuerpo (el encuentro del sujeto con el objeto), que hace referencia a la aparición de los signos y síntomas de la enfermedad; b) la explicación del wixárika a su síntoma (la interacción del sujeto con el objeto), se refiere al momento en que tuvieron conciencia de su enfermedad y sus primeras explicaciones a lo que experimenta en el cuerpo; c) las explicaciones del espacio 
social a su enfermedad (el sujeto y objeto enmarcados en un contexto), que alude al análisis de las interacciones existentes entre el wixárika con el síntoma de enfermedad y su contexto social, para la búsqueda de una explicación lógica a lo que experimenta físicamente.

\section{Los síntomas en el cuerpo}

Al no poder definir la diabetes, los participantes la describieron por la sintomatología o la causa de la enfermedad: reportaron la necesidad frecuente de orinar, constante sed, resequedad de la boca, boca amarga, dolor de cabeza, entumecimiento de alguna extremidad de su cuerpo, imposibilidad de conciliar el sueño, vista borrosa, inflamación de los pies y agotamiento general; como se muestra en los siguientes discursos:

...pos que era dolor de cabeza, con la boca seca, con muchas ganas de tomar agua y en la noche, pos me levantaba a hacer del baño varias veces y después que hacía del baño, pos me daba sed otra vez. (Isela)

...me siento bien, nomás entumido, así no puedo agarrar una cosa se me caen las cosas. (Rosa)

El wixárika se conecta o se relaciona por primera vez con la diabetes a partir de lo que experimenta en el cuerpo: el síntoma físico. Esto, para el psicoanálisis social de PichonRivière $^{(40)}$, se considera como el establecimiento del primer vínculo del wixárika con la diabetes. Las situaciones vividas y experimentadas a través de su cuerpo le han permitido al wixárika contar con una historia vivida con referencia a la diabetes, lo que da paso a una reconfiguración de la subjetividad a partir de la enfermedad. Desde la perspectiva del psicoanálisis social, el objeto -la diabetes-, con el cual establece el primer vínculo el wixárika, se encuentra y actúa en el cuerpo, y constituye al sujeto. Es un vínculo que nace y se conecta dentro del mismo sujeto a partir de la sintomatología física experimentada en el propio cuerpo.

El objeto de la diabetes se encuentra instalado y actúa dentro del cuerpo -en el campo de interacción interno-, por lo que, hasta este momento se han establecido vínculos internos correspondientes al espacio determinado del órgano particular donde el wixárika experimenta los síntomas de la diabetes.

A partir de este encuentro con la diabetes a través del cuerpo del wixárika, se inicia una nueva organización de la subjetividad con la experiencia vivida que le produce el malestar en órganos particulares. Asimismo, desde este momento, y en conjugación con los demás elementos de vida con los que cuenta el wixárika, estará en juego la construcción de un sentido subjetivo que le permitirá conocer lo que le está pasando. En ese primer encuentro se construye una relación entre el wixárika como sujeto y la diabetes como objeto cuyo resultado es la construcción de los vínculos entre sujeto y objeto ${ }^{(40)}$. En esta subjetividad se encuentran articuladas personas, lugares, emociones, pensamientos, con un tiempo propio y expresados a través del establecimiento de vínculos. Todo esto a partir de un proceso de comunicación y aprendizaje entre el wixárika y la diabetes.

\section{La explicación del wixárika a su síntoma}

Los participantes relataron una historia de vida ubicada en un contexto de arduo y excesivo trabajo, así como una alimentación alta en azúcares y carbohidratos, pero donde no se registraba algún padecimiento incapacitante y transgresivo al cuerpo, que limitara su funcionalidad. Por tanto, al percibir los primeros síntomas de la diabetes no encontraban explicación lógica de lo que su cuerpo experimentaba, así que consideraban que los síntomas iban a desaparecer por sí solos y de manera natural y al no desparecer, asumían la idea de una muerte inminente, como lo indican algunos discursos: 
...yo pensaba que se me iba a quitar, pero no se me quitaba entre más, más me iba poniendo malo... por yo, sentía que me iba a morir, así sentía... (Rosa)

...entonces yo sentía que, que pos que ya para mí ya, ya no había vida... (Juventino)

Las experiencias iniciales de los wixáritari con la diabetes, apreciadas desde la lógica de Pichon-Rivière( ${ }^{(4)}$, hablan de cómo el wixárika (sujeto) establece el vínculo con la diabetes (objeto), desde el pensamiento de que los síntomas experimentados en el cuerpo, en un primer momento, iban a desaparecer por sí solos, y cómo, en un segundo momento, al no desparecer, los llevaría a perder la vida. Lo anterior puede evidenciar la dificultad que experimentan los wixáritari para identificar una enfermedad crónica, con las implicaciones que conlleva; en su concepto, solo cuando el síntoma llega a la conciencia, se asumen como enfermos. El primer vínculo se produce, sobre todo, en el cuerpo (área 2) y, posteriormente, el vínculo pasa a la mente (área 1) cuando trata de explicárselo. No obstante, dado que existe una interacción continua con la familia y su entorno, también se manifiesta el vínculo con el mundo externo (área 3) que, en ocasiones, los favorece a sobrellevar los malestares asociados a la diabetes y, en otras, no. En este momento, existe una relación dialéctica entre la diabetes como objeto ubicado en el cuerpo (área 2) y en la mente (área 1), y el establecimiento de determinados vínculos en cada uno de los campos internos de interacción, lo que permite entender cómo es vivida la realidad por el wixárika y su reacción particular ante la enfermedad.

Los wixáritari dejaron transcurrir de 3 a 5 años desde que el objeto (diabetes) se manifestara en algunos de los órganos del cuerpo hasta instrumentar una conducta operativa para cuidar de su salud. Durante este tiempo, solo existió un proceso dialéctico entre lo que experimentaban en el cuerpo y las primeras ideas que aparecieron en sus mentes. Por tanto, de allí en adelante construyeron un ECRO dinámico que, de manera permanente, se fue integrando con nuevos elementos frente a esta relación que tiene el wixárika con la enfermedad. El ECRO que fue construyendo el wixárika sobre la diabetes en cada momento de la experiencia vivida, se fue rectificando y enriqueciendo para lograr una mejor comprensión de los síntomas que se experimentaba en el cuerpo y sus acciones al respecto.

\section{Las explicaciones del espacio social a su enfermedad}

Ante el impacto emocional que produce la idea de que "se van a morir", el síntoma transgrede hasta llegar a la incapacidad. Al "no poderse mover", el wixárika, que no comprende lo que le pasa, busca una explicación a lo que le está sucediendo a su cuerpo (área 2) y esto lo mueve a buscar apoyo en el área 3 (mundo externo ${ }^{(40)}$, representada por el médico alópata del centro de salud; por lo que comienza a instrumentar conductas operativas dirigidas a cuidar su salud. Aquí un discurso que da cuenta de ello:
Entrevistador: ¿y por qué decidiste venir a la clínica?
Isela: no pos porque quería hora, o sea vine pos porque quería saber, saber a ver qué me iba a decir la dotora, por qué tenía esos síntomas...

La entrevista sobre el malestar físico y las pruebas clínicas a partir del análisis de sangre, fue la manera en que los entrevistados reportaron que se les realizó el diagnóstico de diabetes, después de 3 a 5 años de manifestarse los síntomas. En ese momento, obtuvieron una explicación por parte del personal de salud, quienes les informaron que presentaban un exceso de azúcar en la sangre. De esta manera, comienzan a construir referencias en torno al objeto de diabetes. Una de las entrevistadas expresa así su experiencia:

\footnotetext{
...en siguiente año cayó ese doctor, ese sí era muy bueno, y ya me estuvo pregunte
} 
y pregunte y esto y esto y esto... Ah, bueno, mañana en la mañana te vienes en ayunas -me dijo- y ya otro día ya me fui, pero ya estaba muy avanzado de mi enfermedad y ya me fui y ya me sacó la sangre y ya... No, pos tienes mucha azúcar-dice-, ¿desde cuándo has estado así? No pos ya voy casi como tres años [...] cómo iba a saber yo que tenía -le decía yo-, no pues tienes azúcar, dice, y hora vas estar viniendo... (Fátima)

El discurso anterior corresponde a lo que, según Pichon-Rivière ${ }^{(40,43)}$, constituye un vínculo externo con la diabetes. Este vínculo contiene elementos sobre la forma en que el médico de la Secretaría de Salud intenta dar a conocer el malestar físico del wixárika. Aquí encontramos que el espacio social (área 3: mundo externo) brinda una explicación a la experiencia vivida del wixárika con la diabetes y a su causa. De este modo, el espacio social caracterizado por la presencia del personal médico de la Secretaría de Salud incide en la configuración social sobre la enfermedad. No obstante, en un primer momento, el wixárika se encuentra con un ECRO externo, que no asocia con su experiencia personal, por lo que tiende a desconfiar de ello, a negar que él lo padece y a mencionar que "eso dicen los doctores"... aunque en ese encuentro empiezan a identificar que los médicos Ilaman diabetes a los malestares que él ha descrito. Paulatinamente, al platicar con otros diabéticos de su propio pueblo, el wixárika va encontrando ecos de los discursos médicos en su propia vivencia, como se aprecia en los siguientes discursos:

...antes, hace mucho, cuando empecé, dicen -oigo pues 'ora las pláticas-, que pega di [de] un coraje, un susto, que di [de] un no falta que, y yo me vino pegando con, en un coraje... (Juventino).

...pos ella me dice [habla de la doctora], que esa enfermedad puede llegar de, cómo te diré, de un susto o de coraje que haga, o de cosas que te digan que no, que nada más tú escuches y no digas lo que tú sientes, yo en mi caso me imagino que eso fue lo que me hizo daño... (Isela)

De este modo, al escuchar de boca de otros wixaritari un mensaje similar al del médico, el paciente comprende que el susto o el coraje detonan la diabetes, y esto se convierte en un nuevo vínculo que se establece con la enfermedad, lo que le facilita la aceptación del discurso médico. Tal explicación encontrada en el mundo externo (Secretaría de Salud) está en interacción con el mundo interno (cuerpo y mente del wixárika) ${ }^{(40,43)}$ en la búsqueda de la comprensión del malestar que se experimenta en el cuerpo. De este modo, el wixárika intenta integrar el discurso prevaleciente en el mundo externo (área 3) a su mente y cuerpo (área 1 y 2) para comprender lo que le pasa.

El wixárika valida esta explicación sobre la causa de la diabetes, que es proporcionada por el médico de la Secretaría de Salud o algún miembro de la comunidad, a partir de su propia experiencia. La validación se realiza mediante la búsqueda y encuentro de un suceso que se experimentó como susto o coraje. Aluden a sucesos de homicidios, suicidios, demandas, acusaciones legales por violación, golpes físicos de la pareja y problemas de terrenos, que coincidieron con la aparición del malestar físico, por tanto, demuestran para los entrevistados que el susto o coraje causan la diabetes, tal como se lo explicó el personal de salud alópata. Uno de los relatos alusivos, es el siguiente:

..."pos sabes que quiero que jah sí!" -le dijo el presidente-, "sabes que quiero que me entregues tu potrero y todo porque...", y el Armando y ese mentado Raúl, ya, pos ya están apalabrados, desde tiempo que le estaba diciendo que les iban a entregar los potreros [...] Eso oyía yo, y ese día allí salieron y ya dijo, le dijo al que taba rentando: "y sabes que don Juventino y ya queremos el potrero tuyo dijo y lo de julano porque aquí viene un señor ya a recebirlo" [...] ahi ese rato como me dio coraje, pasaron los dos así junto a mí y a risa y risa y diciendo 
todo eso, y pero pos ni modo de que le hubiera dicho eso, dicho sabes, que yo ocupo el potrero, "y te vo a dar tanto de plazo y pa que saques tu ganado, anque no me pagues ehh", ese, es era su modo de dicir pa que quedara acomodado los otros compañeros y de ahi me pegó... sentía muucho coraje, hijo de la chinga y lo pos que se pasaron burlándose del junto a mí... Ey, quisiera... [ha]bía traído una pinche pistola horita los cabronaba, así me, y mira de ahi me pegó un sequío [sed] hijo de la chingada y estaba una señora así, vendiendo raspados [...] por, por este mes de febrero, fue cuando fuimos ya de por sí hace mucha calor en Bolaños, no pos empecé a sentir un sequío y yo, no pos sabe, vuelta y vuelta po allá en una orilla, no pos sabe taba pues no había gente iba y cada rato iba a orinar, bueno pos hora qué tengo, pensé yo entre mí y ya salieron por allí, más temprano que horita, salieron, ya nos juimos nos bian dado una casa pa dormir allí y yo orine, orine, orine hijo de la chin, cada rato pasó allí como veinte minutos, y me preguntó mi tío, ¿pos lo que tienes que cada rato vas al baño? (Juventino)

En el discurso se observa cómo este primer encuentro dialéctico de vínculos establecidos desde el cuerpo (área 2), la mente (área 1) y el mundo externo (área 3) con la diabetes (objeto), como lo menciona ${ }^{(40,44)}$, no es un proceso lineal ni armónico, sino todo lo contrario, está lleno de afirmaciones y negaciones, de encuentros y desencuentros. Es un proceso dialéctico confrontativo y doloroso, en el que se van afirmando saberes, pero también se van negando, en una lógica dialéctica, hasta llegar a una ruptura. La Secretaría de Salud afirma que aquello que experimenta el wixárika en el cuerpo es la diabetes, y continúa enunciando la sintomatología característica de dicha enfermedad. Pero de los relatos surge que la causa desencadenante de esta sintomatología es un susto o un coraje experimentados en algún momento de la historia de vida del wixárika.
El wixárika, con la información que obtiene de su contexto social, enfrenta un conflicto que le permite afinar y rectificar el ECRO que ha empezado a construir. Sin embargo, en este momento, aún no ha logrado integrarlo a su subjetividad (entendida como una totalidad que integra el cuerpo, la mente y el mundo externo); solo se encuentra este esquema conceptual, referencial y operativo en el mundo externo, que propicia la consolidación del proceso dialéctico como proceso creador de la subjetividad del wixárika frente a la diabetes, lo que le permite generar una respuesta adaptativa a la experiencia vivida de la enfermedad. Hasta este momento -después de 3 a 5 años con la enfermedad- el wixárika (sujeto) ha construido un ECRO sobre la diabetes a partir de las relaciones dialécticas que establece la enfermedad, y que necesita afinar y consolidar para presentar una conducta operativa dirigida a la adherencia del tratamiento médico.

\section{DISCUSIÓN}

La diabetes no existe en la cosmovisión, ni en el desarrollo histórico, social y cultural el pueblo wixárika. Es una enfermedad que no les pertenece, ellos consideran que "fue traída por los españoles y no se encuentra dentro de las originadas por la sierra", lo cual remite a la colonización histórica cuyas consecuencias siguen siendo vigentes desde conceptos hegemónicos urbano-occidentales que rigen las prácticas cotidianas de los pueblos. Sin embargo, hoy en día, los wixáritari están padeciendo esta enfermedad crónico-degenerativa como resultado de la adopción acrítica de estilos de alimentación, modos de vida y subsistencia de los mestizos, lo cual se ha vuelto inevitable debido a la gran cercanía geográfica y, en su caso, al acceso cada vez más fácil entre los indígenas y los mestizos, pero también a causa de la discriminación que sufren por pertenecer a un pueblo originario. Por lo tanto, el wixárika (sujeto), al entrar en contacto con la diabetes (objeto), no cuenta con un ECRO necesario 
para tener un abordaje sobre el proceso de salud-enfermedad de la enfermedad que le permita cuidar su salud.

El wixárika, en el primer encuentro con la enfermedad, no logra relacionar factores tales como: estilo de vida, alimentación y genética, con el desarrollo de enfermedades crónicas no transmisibles como la diabetes, por lo que se ve obligado a conocer la perspectiva médica para la comprensión y la intervención de la enfermedad con la finalidad de cuidar su salud. Mientras no suceda esto, el wixárika no podrá comprender que ciertos estilos de vida y alimentación contribuyen al desarrollo de enfermedades como la diabetes, lo que tendrá graves consecuencias para su salud.

El wixárika, en el primer momento de su vida con diabetes, establece vínculos que constituyen un ECRO débil sobre la diabetes, lo que le permite solo la comprensión de la enfermedad, sin generar aún un referente y una conducta sólidos que vayan dirigidas al cuidado de su salud. El ECRO se irá rectificando constantemente a partir de nuevas experiencias que se generan en un tiempo y espacio específicos en una interacción dialéctica entre el cuerpo, la mente y el mundo externo $^{(40)} y$ en un ir y venir a la memoria. De esta manera, se construye una visión totalizadora de la diabetes, a partir de la relación entre dualidades: individuo-sociedad, individuo-organismo, afuera-adentro, útil para el abordaje del proceso de salud-enfermedad, en la que el wixárika genera un nuevo conocimiento sobre la diabetes, enfermedad que no pertenecía a su cultura y que ahora se encuentra instalada en su cuerpo y en su comunidad.

El proceso dialéctico mediante el cual se construye un nuevo ECRO en el wixárika comienza con la tesis de los malestares que experimenta en su cuerpo y la explicación que se da a sí mismo al respecto. Al acudir al mundo externo en busca de respuestas, aparece la antítesis, cuando el wixárika niega la validez de la afirmación que recibe del personal de la Secretaría de Salud acerca de que es diabetes lo que experimenta en el cuerpo. No obstante, surge el tercer momento dialéctico (la síntesis) cuando el wixárika acepta el discurso del médico al contrastarlo con su experiencia personal y entonces consolida la parte conceptual del ECRO, aunque no sin un proceso de ruptura interior y conflicto consigo mismo. $\mathrm{Y}$, como se menciona desde el psicoanálisis social, ahí es donde se encuentra la dialéctica entre los vínculos internos y externos establecidos entre la mente, el cuerpo y el exterior, y este entramado de vínculos permiten la construcción del esquema conceptual, referencial y operativo del wixárika sobre la diabetes.

Las primeras experiencias que manifestaron los wixáritari con la diabetes, respecto de los síntomas en el cuerpo, coinciden con las experiencias reportadas por las mujeres mi'kmaq ${ }^{(39),}$ y los nativos del Tratado 6, Tratado 7, y el Tratado $8^{(13)}$, en Alberta; las metis: saulteaux o forma Ojib- (anishinaabe), -Cree (nêhinaw) y oji-cree ${ }^{(38)}$, en Canadá; nativos originarios en Australia ${ }^{(26,37)}$; los beduinos, en Israel ${ }^{(36)}$; los indios estadounidenses, en EEUU ${ }^{(32-33)}$; y los tzotziles y tzelzales, en México ${ }^{(34)}$. Sin embargo, difieren con las primeras explicaciones que los wixáritari manifestaron sobre los síntomas de la diabetes y con el tiempo en encontrar una explicación a la diabetes: la idea de que desaparecerían solos, el pensamiento de muerte, y el transcurso de un periodo de 3 a 5 años entre estas explicaciones iniciales y el acudir al centro de salud para buscar respuestas.

La explicación sobre la causa de la diabetes que manifiesta el wixárika coincide con lo reportado por los tzotziles y tzeltzales de San Cristóbal de Las Casas y cabeceras municipales de Chamula y Tenejapa, del estado de Chiapas, en México ${ }^{(34)}$; por los diné, hopi, mayan, oglala lakota, yoeme (yaqui) y choctaw de las Primeras Naciones de América del Norte ${ }^{(33)}$; por los mayas en Guatemala ${ }^{(35)}$, y por los apache, akimel o'odham, arikara, assiniboine, cherokee, chippewa, choctaw, covelo, dakota, ho chunk, lakota, menominee, meskwaki, micmac, navajo, odawa, ojibwe, omaha, oneida, ponca, potawatomi, pueblo, sac y fox, seneca, sioux y stockbridge de las Primeras Naciones de América del Norte, de Canadá( ${ }^{(32)}$. Reconocen 
que el desencadenamiento de la diabetes se liga a un suceso crítico en el cual se ha producido bastante sufrimiento y que, a la par de ese suceso, se manifiestan los síntomas de la diabetes. Es importante reconocer, que los participantes reportados en los anteriores estudios son considerados indígenas "urbanos" y esta circunstancia da cuenta del grado de occidentalización del cual parten estos nativos para la comprensión y explicación de la diabetes. Mientras que su comprensión sobre la causa de la enfermedad integra tanto el evento de susto o coraje, acompañado de sufrimiento, como la relación entre la biología humana y la alimentación y su consecuencia con el desarrollo de la diabetes ${ }^{(35)}$, los wixáritari no relacionan la biología, el estilo de vida y la alimentación con su impacto en los procesos de salud-enfermedad.

La principal fortaleza de esta investigación reside en el método y fundamento teórico, porque permitieron la comprensión de la subjetividad que construye el wixárika a razón de la experiencia con la diabetes meIlitus tipo 2.
Los vínculos que establece el wixárika que padece diabetes mellitus tipo 2 con su enfermedad permiten comprender las tensiones, los conflictos y las resistencias que se manifiestan en la experiencia vivida con la diabetes, para llegar a presentar conductas dirigidas a recuperar la salud, en el contexto específico de la cultura wixárika. El estudio evidencia que las intervenciones en el tratamiento y prevención de la diabetes debe considerar, además de lo médico-biológico, los aspectos psicológico, social y cultural, para lograr eficacia en la adherencia a los tratamientos. De no hacerlo, las intervenciones y tratamientos fallarán y pondrán en riesgo la existencia de la comunidad wixárika. Además, a partir de estos resultados, se pueden abrir otras líneas de investigación que permitan comprender mejor la apreciación sobre la diabetes en la comunidad, considerando otros actores, tales como los mara'kates, los familiares, las autoridades tradicionales y el personal de salud occidental.

\section{AGRADECIMIENTOS}

A las autoridades tradicionales de la comunidad Mesa del Tirador y a las autoridades de la Secretaría de Salud de Jalisco, México, por el apoyo recibido para realizar la investigación. Agradecemos el financiamiento recibido por Kellogg Company México, S de R. L. de C. V., para el desarrollo de la investigación, a través del programa "Apoyo a proyectos de investigación en nutrición (APIN): convocatoria 2015".

\section{REFERENCIAS BIBLIOGRÁFICAS}

1. Organización Mundial de la Salud. Informe mundial sobre la diabetes [Internet]. Ginebra:
OMS; 2016 [citado 1 abr 2018]. Disponible en: https://tinyurl.com/y7cxpqtu.

2. De la Torre García R, (coord.). Informe sobre desarrollo humano de los pueblos indígenas en México: El reto de la desigualdad de oportunidades [Internet]. México DF: PNUD; 2010 [citado 1 abr 2018]. Disponible en: https://tinyurl.com/ y7voeaxb.

3. Secretaría de Salud. Sistema de Vigilancia Epidemiológica: Casos nuevos de enfermedades en el periodo 2008-2013 de la Región Sanitaria Norte I de Jalisco México. Jalisco: Secretaría de Salud; 2013.

4. Secretaría de Salud. Protocolo clínico para el diagnóstico y tratamiento de la diabetes [Internet]. 
México DF: Secretaría de Salud; 2015 [citado 1 abr 2018]. Disponible en: https://tinyurl.com/y9tpdrqx.

5. Instituto Nacional de Estadística y Geografía. Encuesta Intercensal 2015: Base de Datos Jalisco [Internet]. México: INEGl; 2015 [citado 5 ene 2018]. Disponible en: https://tinyurl.com/y8sk4y9r.

6. Webster E, Johnson C, Kemp B, Smith V, Johnson $\mathrm{M}$, Townsend $\mathrm{B}$. Theory that explains an Aboriginal perspective of learning to understand and manage diabetes. Australian and New Zealand Journal of Public Health. 2017;41(1):27-31. Disponible en: doi: 10.1111/1753-6405.12605.

7. Dowse GK, Spark RA, Mavo B, Hodge AM, Erasmus RT, Gwalimu M, Knight LT, Koki G, Zimmet PZ. Extraordinary prevalence of noninsulin-dependent diabetes mellitus and bimodal plasma glucose distribution in the Wanigela people of Papua New Guinea. The Medical Journal of Australia.1994;160(12):767-774.

8. Taylor R, Jalaludin B, Levy S, Montaville B, Gee $K$, Sladden T. Prevalence of diabetes, hypertension and obesity at different levels of urbanisation in Vanuatu. The Medical Journal of Australia. 1991;155(2):86-90.

9. Franco LJ. Diabetes in Brazil: a review of recent survey data. Ethnicity \& Disease. 1992;2(2):158-165.

10. Manitowabi D, Maar M. Coping with colonization: Aboriginal diabetes on manitoulin Island. En: Fear-Segal J, Tillett R, (eds.). Indigenous bodies: reviewing, relocating, reclaiming. New York: State University of New York Press; 2013. p. 145-159.

11. Johnson JA, Vermeulen SU, Hugel G, Toth EL, Hemmelgarn BR, Ralph-Campbell K, King M. Increasing incidence and prevalence of diabetes among the Status Aboriginal population in urban and rural Alberta 1995 to 2006. Canadian Journal of Diabetes. 2008;32(4):343. doi: 10.1016/S14992671(08)24172-9.

12. Leal Ferreira M, Lang GC, (eds.). Indigenous peoples and diabetes: community empowerment and wellness. Durham: Carolina Academic Press; 2006.

13. Oster RT, Grier A, Lightning R, Mayan MJ, Toth EL. Cultural continuity, traditional Indigenous language, and diabetes in Alberta First Nations: a mixed methods study. International Journal for Equity in Health. 2014;13(1):92-103. doi: 10.1186/s12939-014-0092-4.

14. Saumade F. De la sangre al oro: la transubstanciación del cristianismo y del capitalismo en la comida ritual huichol (México). Amérique Latine
Histoire et Mémoire-Les Cahiers ALHIM [Internet]. 2013;25. Disponible en: https://journals.openedition.org/alhim/4618.

15. Saumade F. Toro, venado, maíz, peyote: El cuadrante de la cultura wixárika. Revista de El Colegio de San Luis. 2013;3(5):16-54.

16. Medina Miranda HM. Las personalidades del maíz en la mitología wixárika o cómo las mazorcas de los ancestros se transformaron en peyotes. Revista de El Colegio de San Luis. 2013;3(5):164-184.

17. Fajardo Santana H. Comer y dar de comer a los dioses; Terapéuticas en encuentro: Conocimiento, proyectos y nutrición en la Sierra Huichola. México DF: Lagos de Moreno, Universidad de Guadalajara, El Colegio de San Luis; 2007.

18. Guzmán-Mejía R, del Carmen Anaya-Corona M. Cultura de Maíz-Peyote-Venado: Sustentabilidad del pueblo Wixarika. 1a ed. México DF: Universidad de Guadalajara; 2007.

19. Comisión Nacional para el Desarrollo de los Pueblos Indígenas. Acciones de Gobierno para el Desarrollo Integral de los Pueblos Indígenas. 1a ed. México DF: Comisión Nacional para el Desarrollo de los Pueblos Indígenas; 2011.

20. Benítez F. Los indios de México. Vol. 2. México DF: Ediciones Era; 1968.

21. Torres JJ. El hostigamiento a "el costumbre" huichol: los procesos de hibridación social. México DF: El Colegio de Michoacán, Universidad de Guadalajara; 2000.

22. Neurath J. Huicholes: Pueblos indígenas del México contemporáneo [Internet]. México DF: PNUD; 2003 [citado 5 ene 2018]. Disponible en: https://tinyurl.com/yaton56w.

23. Zingg RM. Los huicholes: una tribu de artistas. T. 1. México DF: Instituto Nacional Indigenista; 1982.

24. Secretaría de Salud. Unidades de atención médica en las comunidades wixáritari en el norte de Jalisco México. Jalisco: Secretaría de Salud; 2013.

25. Arrivillaga M, Correa D, Salazar IC, (eds.). Psicología de la salud: abordaje integral de la enfermedad crónica. Bogotá: Manual Moderno; 2007.

26. Aspin C, Brown N, Jowsey T, Yen L, Leeder S. Strategic approaches to enhanced health service delivery for Aboriginal and Torres Strait Islander people with chronic illness: a qualitative study. BMC Health Services Research. 2012;12(1):143- 
152. doi: 10.1186/1472-6963-12-143.

27. Cerón A, Ruano AL, Sánchez S, Chew AS, Díaz D, Hernández A, Flores W. Abuse and discrimination towards indigenous people in public health care facilities: experiences from rural Guatemala. International Journal for Equity in Health. 2016;15(1):77-84. doi: 10.1186/s12939-016-0367-z.

28. Van Herk KA, Smith D, Andrew C. Identity matters: Aboriginal mothers' experiences of accessing health care. Contemporary Nurse. 2010;37(1):57-68. doi: 10.5172/ conu.2011.37.1.057.

29. Durey A, Thompson SC. Reducing the health disparities of Indigenous Australians: time to change focus. BMC Health Services Research. 2012; 12(1):151-162. doi: 10.1186/1472-6963-12-151.

30. Dyck RF, Karunanayake C, Janzen B, Lawson J, Ramsden VR, Rennie DC, Gardipy PJ, McCallum $\mathrm{L}$, et al. Do discrimination, residential school attendance and cultural disruption add to individuallevel diabetes risk among Aboriginal people in Canada? BMC Public Health. 2015;15(1):12221233. doi: 10.1186/s12889-015-2551-2.

31. Saforcada E, Lellis M, Mozobancyk S. Psicología y salud pública: nuevos aportes desde la perspectiva del factor humano. Buenos Aires: Paidos; 2010.

32. Pollak ME. Diabetes in Native Chicago: An ethnography of identity, community, and care [Internet]. [Tesis de doctorado]. Madison: The University of Wisconsin; 2015 [citado 5 ene 2018]. Disponible en: https://tinyurl.com/y9cmthv9.

33. Sanderson PR, Little $M$, Vasquez $M$, Lomadafkie B, Brings Him Back-Janis M, Trujillo V, Jarratt-Snider K, Teufel-Shone NI, et al. A perspective on diabetes from indigenous views. Fourth World Journal. 2012;11(2):57-78.

34. Page Pliego JT. Refresco y diabetes entre los mayas de Tenejapa, San Cristóbal de Las Casas y Chamula, Chiapas. LiminaR Estudios Sociales y Humanísticos. 2013;11(1):118-133. doi: 10.29043/liminar.v11i1.102.

35. Little M. Type 2 diabetes in rural Guatemala: disease perceptions, service-provision difficulties and management techniques. Undercurrent. 2012;9(1):25-35.

36. Alshamari Abu Nadi F. Health inequalities and the right to healthcare of Negev Bedouin in Israel with diabetes: A case study of a marginalized Arab indigenous minority [Internet]. [Tesis de doctorado]. Coventry: University of Warwick;
2013 [citado 5 ene 2018]. Disponible en: http:// wrap.warwick.ac.uk/59612/.

37. Cuesta-Briand B, Saggers S, McManus A. "You get the quickest and the cheapest stuff you can": food security issues among low-income earners living with diabetes. The Australasian Medical Journal. 2011;4(12):683-691. doi: 10.4066/AMJ.20111104.

38. Tait Neufeld H. Patient and caregiver perspectives of health provision practices for First Nations and Metis women with gestational diabetes meIlitus accessing care in Winnipeg, Manitoba. BMC Health Services Research. 2014;14:440-454. doi: 10.1186/1472-6963-14-440.

39. Whitty-Rogers JP. Exploring Mi'kmaq women's experiences with gestational diabetes mellitus [Internet]. Edmonton: University of Alberta; 2013 [citado 5 ene 2018]. Disponible en: https://tinyurl. com/y8wx7vnm.

40. Pichon-Rivière E. Teoría del vínculo. Buenos Aires: Nueva Visión; 1985.

41. Good BJ. Phenomenology, psychoanalysis, and subjectivity in java. Ethos. 2012;40(1):24-36. doi: 10.1111/j.1548-1352.2011.01229.x.

42. Good BJ. Theorizing the "subject" of medical and psychiatric anthropology. Journal of the Royal Anthropological Institute. 2012;18(3):515-535. doi: 10.1111/j.1467-9655.2012.01774.x.

43. Pichon-Rivière E. El proceso creador: Del psiconálisis a la psicología social III. Buenos Aires: Nueva Visión; 1987.

44. Pichon-Rivière E. El proceso grupal: Del psicoanálisis a la psicología social I. Buenos Aires: Nueva Visión; 1985.

45. Girardi IC. Ética en investigación cualitativa. En: Estrategias de investigación cualitativa en psicología y educación. México DF: Universidad Intercontinental; 2011. p. 307-315.

46. Merleau-Ponty M. Fenomenología de la percepción. México DF: Fondo de Cultura Económica; 1975.

47. Ponty MM, González IB, Piérola AR. La fenomenología y las ciencias del hombre. Buenos Aires: Novoa; 1964.

48. Merleau-Ponty M, Fisher AL. The structure of behavior. Boston: Beacon Press; 1963.

49. Merleau-Ponty M. Sentido y sinsentido. Barcelona: Península; 1977. 
50. Martínez-Salgado C. El muestreo en investigación cualitativa: Principios básicos y algunas controversias. Ciência \& Saúde Coletiva. 2012;17(3):613-619. doi: 10.1590/S1413-812320 12000300006 .

51. Navarrete JM. El muestreo en la investigación cualitativa [Internet]. Investigaciones Sociales. 2000 [citado 5 ene 2018];4(5):165-180. Disponible en: https://tinyurl.com/ybfs4mtk.

52. Flick U. Introducción a la investigación cualitativa. 2a ed. Madrid: Morata; 2012.

53. Marradi A, Archenti N, Piovani JI. Metodología de las ciencias sociales. Buenos Aires: Cenage Learning; 2007.

54. Taylor SJ, Bogdan R. Introducción a los métodos cualitativos de investigación. Barcelona: Paidós; 1987.
55. Bajtín M. Estética de la creación verbal. México DF: Siglo XXI Editores; 2012.

56. Bajtín M. Los géneros discursivos en la estética de la creación verbal. Buenos Aires: Siglo XXI Editores; 1985.

57. ATLAS.ti. Berlín: Scientific Software Development; c2002-2019.

58. México. Reglamento de la ley general de salud en materia de investigación para la salud en México de 1987 [Internet]. Diario Oficinal de la Federación; 2 mar 2014 [citado 15 ene 2018]. Disponible en: https://tinyurl.com/y9xww84c.

59. Sociedad Mexicana de Psicología. Código ético del psicólogo. México DF: Trillas; 2010.

\section{FORMA DE CITAR}

Quiñonez-Tapia F, Vargas-Garduño ML, Soltero-Avelar R. Los wixáritari con diabetes mellitus y sus vínculos con la enfermedad: desde la aparición del síntoma hasta una primera explicación. Salud Colectiva. 2019;15:e1856. doi: 10.18294/sc.2019.1856.

Recibido: 24 de abril de 2018 | Versión final: 28 de noviembre de 2018 | Aprobado: 3 de diciembre de 2018

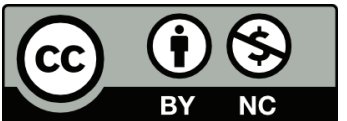

Esta obra está bajo una licencia de Creative Commons Reconocimiento-NoComercial 4.0 Internacional. Reconocimiento - Permite copiar, distribuir y comunicar públicamente la obra.

A cambio, se debe reconocer y citar al autor original. No Comercial - Esta obra no puede ser

utilizada con finalidades comerciales, a menos que se obtenga el permiso. 